\title{
Caries de la infancia temprana en población de 3 a 5 años de edad
}

\section{Early childhood caries in the population of three to five years of age}

DOI: $10.46981 /$ sfjhv2n3-006

Received in: April 1st, 2021

Accepted in: May 31th, 2021

\author{
M. M. S. Laura Patricia Sáenz Martínez \\ Universidad Autónoma Metropolitana Xochimilco \\ Correo: lpsaenz@correo.xoc.uam.mx \\ Dra. Ma. del Carmen Osorno Escareño \\ Universidad Autónoma Metropolitana Xochimilco \\ Dra. Leonor Sánchez Pérez \\ Universidad Autónoma Metropolitana Xochimilco \\ Dra. Ma. Isabel Luengas Aguirre \\ Universidad Autónoma Metropolitana Xochimilco
}

\section{RESUMEN}

La caries de la infancia temprana (CIT) se desarrolla poco después del inicio de la erupción de los primeros dientes temporales, evoluciona rápidamente e implica un riesgo mayor de nuevas lesiones y atención de urgencias.

Objetivo. Estimar la prevalencia de CIT en preescolares entre 3 y 5 años de edad, al sur de la ciudad de México.

Metodología. Estudio transversal y descriptivo, con una muestra por conveniencia de niños que asistían a una escuela de educación preescolar. Se conformó por 136 infantes (82 niñas y 54 niños), previo consentimiento informado de los padres, cuya edad promedio fue de $4.3 \pm 0.8$ años. El diagnóstico clínico de la CIT lo realizaron dos examinadores calibrados (Kappa 0.89, $\mathrm{P}<0.001$ ) con criterios de la OMS. El análisis se realizó en el programa SPSS y consistió en medidas de tendencia central, dispersión y $\mathrm{X}^{2}$ $(\mathrm{P}<0.05)$.

Resultados. La distribución de la población por género y edad no mostró diferencias significativas ( $\mathrm{P}>0.124)$. La presencia de CIT afectó a $63.4 \%$ de niñas y $61.1 \%$ de niños sin diferencias ( $\mathrm{P}=0.786)$. La prevalencia de CIT a los 3 años fue de $40.7 \%$, a los 4 años de $78.9 \%$ y a los 5 años de $62 \%$ ( $\mathrm{P}=0.007$ ). Los incisivos centrales superiores tuvieron el mayor número de superficies cariadas, seguidos por los primeros molares y los dientes menos afectados fueron los laterales inferiores. La superficie oclusal/incisal fue la de mayor presencia de caries.

Conclusiones. La prevalencia de CIT fue alta en esta región de la ciudad y los dientes más afectados fueron los centrales superiores.

Palabras clave. Caries de la infancia temprana, preescolares, dentición temporal, prevalencia.

\section{ABSTRACT}

Early childhood caries (ECC) is developed a little after the beginning of the eruption of the first temporary teeth, it evolves rapidly and implicates a major risk of new lesions and urgent attention.

Objective. To estimate the prevalence of ECC in pre-school students between three to five years of age, in the south of Mexico City. 
Methodology. Transversal and descriptive study, with a convenience sample of pre-school children. It was formed by 136 infants ( 82 girls and 54 boys), with previous informed consent from the parents, and whose average age was of $4.3 \pm 0.8$ years. The clinical diagnose of the ECC was performed by two calibrated examiners (Kappa 0.89, $\mathrm{P}<0.001$ ) following the WHO criteria. The analysis was made using the SPSS program with central trend measurements, dispersion and $\mathrm{X}^{2}(\mathrm{P}<0.05)$.

Results. The distribution of the population by gender and age did not show any significance differences ( $\mathrm{P}>0.124)$. The presence of ECC affected $63.4 \%$ of the girls and $61.1 \%$ of the boys with no differences $(\mathrm{P}=0.786)$. The prevalence of ECC at three years of age was of $40.7 \%$, at four of $78.9 \%$ and at five of $62 \%(\mathrm{P}=0.007)$.

The central superior incisor teeth had the highest number of surfaces with cavities, followed by the first molars, and the inferior lateral teeth were the least affected. The oclusal/incisal surface was the one that presented more cavities.

Conclusions. The prevalence of ECC was high in this region of the city, and the most affected teeth were the central superior ones.

Key words. Early childhood caries, pre-school students, temporary dentition, prevalence.

\section{INTRODUCCIÓN}

La Organización Mundial de la Salud (OMS) en 2016 declaró que la caries dental era una de las principales morbilidades que afectaban a los niños, por su relación con la salud general durante el crecimiento y el desarrollo (GBD, 2017), así como por las posibles "implicaciones en la calidad de vida de los niños y las niñas (Sheiham, 2006), la cual en gran medida es prevenible o tratable en sus etapas iniciales.

La caries dental es un proceso dinámico en donde existe un equilibrio entre los factores patológicos que causan la pérdida mineral a través del ataque del ácido generado por bacterias cariogénicas (Fejerskov, Nyvad y Kidd, 2015) y por la presencia de factores de protección como la capacidad amortiguadora salival, la exposición al ion flúor que puede remineralizar una lesión incipiente (Kagihara, Niederhauser y Star, 2009) y la resistencia del esmalte a la disolución ácida (Featherstone et al., 2003), también se asocia con factores ambientales, socioculturales, económicos y del comportamiento (Peres et al., 2005; Petersen, 2003).

El inicio de la caries se da poco después de la erupción de los primeros dientes temporales y puede progresar rápidamente y es la enfermedad crónica más común en esta etapa de la vida (Marshall et al., 2003). Dadas estas características y el momento de su aparición, el Centro de Prevención y Control de Enfermedades de Estados Unidos (CDC por sus siglas en inglés) en el año 1994, recomendó el uso del término Early Childhood Caries (ECC) o Caries de Infancia Temprana (CIT) por ser el que mejor refleja la etiología multifactorial de la enfermedad y que afecta a los niños menores de 6 años (American Academy of Pediatric Dentistry, 2008-2009). 
La CIT afecta a un gran número de dientes, probablemente es una de las condiciones más graves en la salud de los infantes, debido a su alta prevalencia y su potencial de incrementar el riesgo a desarrollar caries en la dentición permanente (Achahui et al., 2014).

Una revisión sistemática en 2015 informó que el 9\% de los niños en todo el mundo tenía en sus dientes temporales caries dental no tratada (Kassebaum et al., 2015), debido principalmente al costo económico elevado que, en muchas ocasiones, requería de una restauración extensa, de extracciones dentales a edades tempranas, así como algún tipo de prótesis o mantenedor de espacio, de ahí que la Academia Americana de Odontopediatría (AADP) reconoce que la CIT era un problema de salud pública (AAPD, 2003). Las consecuencias de no tratar este problema de salud pueden llevar a una serie de secuelas como son: infecciones, problemas estéticos, aparición de maloclusiones (Villena, Pachas, Sánchez y Carrasco, 2011), así como su asociación con anomalías cardiacas y endocarditis bacteriana, entre otras (Begzati, Berisha y Meqa, 2010).

La relevancia de la CIT se ha evidenciado en diversas publicaciones tanto en México, así como en América Latina y Europa sobre la salud bucal en niños realizadas en población de 5 y/ó 6 años cuyas prevalencias fueron: Brasil: 46.6\%, (Ministerio da Salud, 2012), Colombia: 62.24\% (Ministerio de Salud, 2014) y Chile: 70.4\% (Ministerio de Salud, 2010), España: 31.5\%, (Bravo et al., 2016), Reino Unido: 37.1\%, (Massod, Mnatzaganian y Baker, 2018).

En México el Programa de Fluoración de la sal de mesa se consolidó en el año de 1992 (Norma Oficial Mexicana 040, 2010) y desde esa fecha se han realizado dos encuestas nacionales de caries, cuyos resultados muestran la disminución de la prevalencia de caries en población escolar y adolescente (Secretaría de Salud, 2001; Secretaría de Salud, 2014). Sobre esta temática existen algunas evidencias científicas en el país que reportan prevalencias de CIT en menores de 6 años con cifras que fluctúan entre 48\% y 76\% (Guerrero, Aguilar, Gutiérrez y Lara, 2015; Rodríguez, Contreras, Arjona, Soto y Alanís, 2006) respectivamente y datos oficiales de 2019 publicaron cifras de CIT en población de 3 a 5 años del 71.4\% (Secretaría de Salud, 2020).

Los datos de salud bucal en población de 6 años publicados en la Encuesta Nacional de Caries y Fluorosis Dental (Secretaría de Salud, 2014) señalan la necesidad de realizar estudios que proporcionen información sólida sobre la presencia de CIT que permita implementar, en etapas tempranas, acciones que privilegien las medidas de 1er nivel de atención sobre todo las que corresponden a los adultos a cargo de los niños. Así, el objetivo de esta investigación fue estimar la prevalencia de CIT en infantes de 3 a 5 años, en una zona sur de la ciudad de México.

\section{MATERIALES Y MÉTODOS}

Tipo de estudio: Transversal, descriptivo y observacional. 
Se obtuvo el permiso de autoridades de la Secretaría de Educación de la Alcaldía de Tláhuac en la ciudad de México, para realizar el levantamiento de la información en una escuela de educación preescolar de la misma demarcación en el turno vespertino. Posteriormente se envió invitación a las madres/padres para dar a conocer los objetivos de la revisión de los niños, así como el beneficio que obtendrían con su participación al conocer la presencia de caries dental de su hija/hijo y, en caso afirmativo, serían informados y remitidos para su atención a una clínica dental de la Universidad Autónoma Metropolitana Xochimilco (UAM-X). El protocolo de estudio fue aprobado por el comité de Ética de la División de Ciencias Biológicas y de la Salud de la UAM-X, con número IRB: CE.2017.002.

Población: Se enviaron 165 consentimientos informados a los padres y se obtuvo el 82\% de respuestas afirmativas $(\mathrm{n}=136)$, por lo que la muestra fue por conveniencia.

Tláhuac es una de las 16 alcaldías que se localiza al sureste de la capital mexicana, con más de $83 \mathrm{~km}^{2}$ dividida en siete pueblos originarios con raíces prehispánicas y es una de la mayores aglomeraciones urbanas en el mundo. La población de Tláhuac en 2020 era de 392,313 personas (51.5\% mujeres y 48.5\% hombres) (INEGI, 2020). El crecimiento demográfico en la alcaldía lo constituyen inmigrantes de otras partes de la ciudad de México, así como de otros estados del país (INEGI, 2011) y su índice de desarrollo humano es de 0.780 (Programa de Naciones Unidad para el Desarrollo, 2015).

Diagnóstico dental: Se utilizó el índice de caries dental por superficie (cpos) el cual se identificó por dos examinadores calibrados con un valor Kappa de $0.89, \mathrm{P}<0.001$, a través de los criterios de la OMS (WHO, 2013), con ayuda de espejo bucal y sonda periodontal tipo “E”. Los datos se analizaron en el programa SPSS (v.25) con medidas de tendencia central, dispersión, para comparar la información de variables cuantitativas se utilizó la prueba $t$ de Student y para las variables cualitativas la prueba de $\mathrm{Chi}^{2}$. Para analizar el comportamiento de los componentes del índice cpos por edad y sexo se aplicó la prueba ANOVA. Se consideró como significativo un valor de $\mathrm{P}<0.005$.

\section{RESULTADOS}

La población estuvo conformada por 82 niñas $(60.3 \%)$ y 54 niños $(39.7 \%)$ con edades entre 37 y 71 meses y un promedio de $4.3 \pm 0.8$ años. Se aprecia en el Cuadro 1, la distribución de la muestra por años cumplidos y sexo cuya distribución fue homogénea $(\mathrm{P}=0.124)$.

Cuadro \#1. Porcentaje de la población según edad y sexo

\begin{tabular}{|c|c|c|c|c|c|c|}
\hline \multirow{3}{*}{$\begin{array}{l}\text { Edad en } \\
\text { años }\end{array}$} & \multicolumn{4}{|c|}{ Sexo } & \multirow{2}{*}{\multicolumn{2}{|c|}{ Total }} \\
\hline & \multicolumn{2}{|c|}{ o } & \multicolumn{2}{|c|}{ 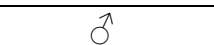 } & & \\
\hline & n & $\%$ & n & $\%$ & $\mathbf{n}$ & $\%$ \\
\hline 3 & 19 & 14.0 & 8 & 5.9 & 27 & 19.9 \\
\hline 4 & 26 & 19.1 & 12 & 8.8 & 38 & 27.9 \\
\hline 5 & 37 & 27.2 & 34 & 25.0 & 71 & 52.2 \\
\hline Total & 82 & 60.3 & 54 & 39.7 & 136 & 100 \\
\hline
\end{tabular}




$$
\uparrow=\text { niñas } \quad \hat{\sigma}=\text { niños } \quad \text { Valor de } \mathrm{P}=0.124 \text {, utilizando la prueba de } \mathrm{Chi}^{2}
$$

Respecto a la presencia de caries en el Cuadro 2 se observa que las niñas presentaron una prevalencia de caries ligeramente superior $63.4 \%$ que los niños $61.1 \%$, sin diferencias significativas en esta distribución.

Cuadro 2. Distribución de niños con y sin caries

\begin{tabular}{|c|c|c|}
\hline SEXO & Sin caries & Con caries \\
\hline Niñas & $36.6 \%$ & $63.4 \%$ \\
\hline Niños & $38.9 \%$ & $61.1 \%$ \\
\hline Total & $37.5 \%$ & $62.5 \%$ \\
\hline \multicolumn{2}{|c|}{ Valor de $\mathrm{P}=0.7879$ con la prueba $\mathrm{dhi}^{2}$}
\end{tabular}

Valor de $\mathrm{P}=0.7879$ con la prueba de $\mathrm{Chi}^{2}$

La CIT se incrementa conforme la edad como se observa en el Cuadro 3, esta distribución fue estadísticamente diferente $(\mathrm{p}=0.007)$, en específico las niñas mostraron diferencia significativa $(\mathrm{p}=0.011)$ datos que no se muestran.

Cuadro 3. Presencia de CIT y su relación con años cumplidos

\begin{tabular}{|c|c|c|c|c|}
\hline \multirow{2}{*}{$\begin{array}{c}\text { Edad en } \\
\text { años }\end{array}$} & \multicolumn{4}{|c|}{ CIT } \\
\cline { 2 - 5 } & $\mathbf{n}$ & $\%$ & $\mathbf{n}$ & $\%$ \\
\cline { 2 - 5 } & & & & \\
\hline 3 & 11 & 8.1 & 16 & 11.8 \\
\hline 4 & 30 & 22.1 & 8 & 5.9 \\
\hline 5 & 44 & 32.4 & 27 & 19.9 \\
\hline Total & 85 & 62.5 & 51 & 37.5 \\
\hline
\end{tabular}

El $37.5 \%$ de los preescolares se encontró libre de caries. El cpos global fue de $8.1 \pm 11.3$ sin diferencias entre sexo $(\mathrm{P}=0.7143)$ y al desglosarlo se observa que a los 3 y 4 años fue mayor en los niños y a los 5 años en las niñas (Cuadro 4). El cpod global fue de 3.5 \pm 3.9 sin diferencia entre edad o sexo (datos que no se muestran).

A la edad de 3 años los componentes del índice cpos fueron mayores en los niños, a los 4 años tuvo un comportamiento similar, excepto en el componente de superficies perdidas que fue ligeramente más alto en las niñas. A los 5 años los mayores valores correspondieron a las niñas en el componente de superficies cariadas, sin diferencias significativas $(\mathrm{P}>0.05)$.

Cuadro 4. Distribución por edad y sexo de los componentes del índice de caries por superficie.

\begin{tabular}{|c|c|c|c|c|c|c|}
\hline edad & Sexo & cs (ds) & ps (ds) & os (ds) & cpos (ds) & $\begin{array}{c}\text { cpos } \\
\text { global }\end{array}$ \\
\hline \multirow{2}{*}{3} & Niñas & $2.2(3.3)$ & - & $0.6(2.3)$ & $2.8(4.5)$ & \multirow{2}{*}{$4.9(11.1)$} \\
\cline { 2 - 6 } & Niños & $6.4(11.0)$ & $1.3(3.6)$ & $2.1(6.0)$ & $9.9(19.1)$ & \\
\hline \multirow{4}{*}{} & Niñas & $6.0(7.3)$ & $0.2(1.0)$ & $1.4(2.9)$ & $7.5(8.3)$ & $8.2(8.7)$ \\
\hline
\end{tabular}




\begin{tabular}{|c|c|c|c|c|c|c|}
\hline & Niños & $7.0(7.1)$ & - & $2.8(4.2)$ & $9.8(9.6)$ & \\
\hline \multirow{2}{*}{5} & Niñas & $7.4(12.2)$ & - & $2.9(7.1)$ & $10.4(13.5)$ & \multirow{2}{*}{$9.2(12.4)$} \\
\cline { 2 - 6 } & Niños & $4.1(5.9)$ & - & $3.7(9.2)$ & $7.8(11.3)$ & \\
\hline
\end{tabular}

cpos $=$ superficies temporales cariadas, perdidas y obturadas; $c s=$ superficies cariadas, $p s=$ superficies perdidas; os $=$ superficies obturadas; valor de P>0.05 por componentes con la prueba ANOVA y t de Student.

La mayor prevalencia de caries fue para los incisivos centrales superiores $(22.7 \%$ los derechos y $20.3 \%$ los izquierdos) y en seguida los primeros molares inferiores izquierdos (15\%) seguidos de los primeros molares superiores izquierdos con 14.1\% (Cuadro 5), sin diferencias en esta distribución $(\mathrm{P}>0.05)$.

Cuadro 5. Prevalencia de caries por grupos de dientes y maxilar

\begin{tabular}{|c|c|c|c|c|}
\hline \multirow{2}{*}{ DIENTE } & \multicolumn{2}{|c|}{ MAXILAR SUPERIOR } & \multicolumn{2}{|c|}{ MAXILAR INFERIOR } \\
\cline { 2 - 5 } & $\begin{array}{c}\text { Derecho } \\
(\boldsymbol{\%})\end{array}$ & $\begin{array}{c}\text { Izquierdo } \\
\mathbf{( \% )}\end{array}$ & $\begin{array}{c}\text { Derecho } \\
(\boldsymbol{\%})\end{array}$ & $\begin{array}{c}\text { Izquierdo } \\
\mathbf{( \% )}\end{array}$ \\
\hline Incisivo central & 22.7 & 20.3 & 3.4 & 3.6 \\
\hline Incisivo lateral & 11.9 & 13.6 & 1.6 & 1.8 \\
\hline Canino & 1.6 & 3.6 & 1.7 & 1.2 \\
\hline Primer molar & 11.8 & 14.1 & 13.9 & 15.0 \\
\hline Segundo molar & 3.1 & 5.4 & 8.8 & 10.0 \\
\hline
\end{tabular}

Valor de $\mathrm{P}>0.05$, con la prueba de $\mathrm{Chi}^{2}$

Ahora bien, la mayor presencia de CIT correspondió al grupo de dientes anteriores y posteriores $(39 \%)$ sin que se observaran diferencias significativas por sexo $(\mathrm{P}=0.578)$. Al desglosar la CIT por grupos de dientes a la edad de 3 años la mayor presencia de caries correspondió a los dientes anteriores, pero a partir de los 4 y 5 años la mayor frecuencia fue para los dientes posteriores. Las mayores expresiones de lesiones de caries se observaron en los niños que tenían afectación tanto en dientes anteriores como posteriores incrementándose conforme al a edad (información que no se muestra).

Al analizar la información por superficie de las superficies de los incisivos las de mayor afectación fueron las superficies mesiales y la de menor afectación fueron las incisales, a excepción de los laterales superiores (Gráfica 1). 


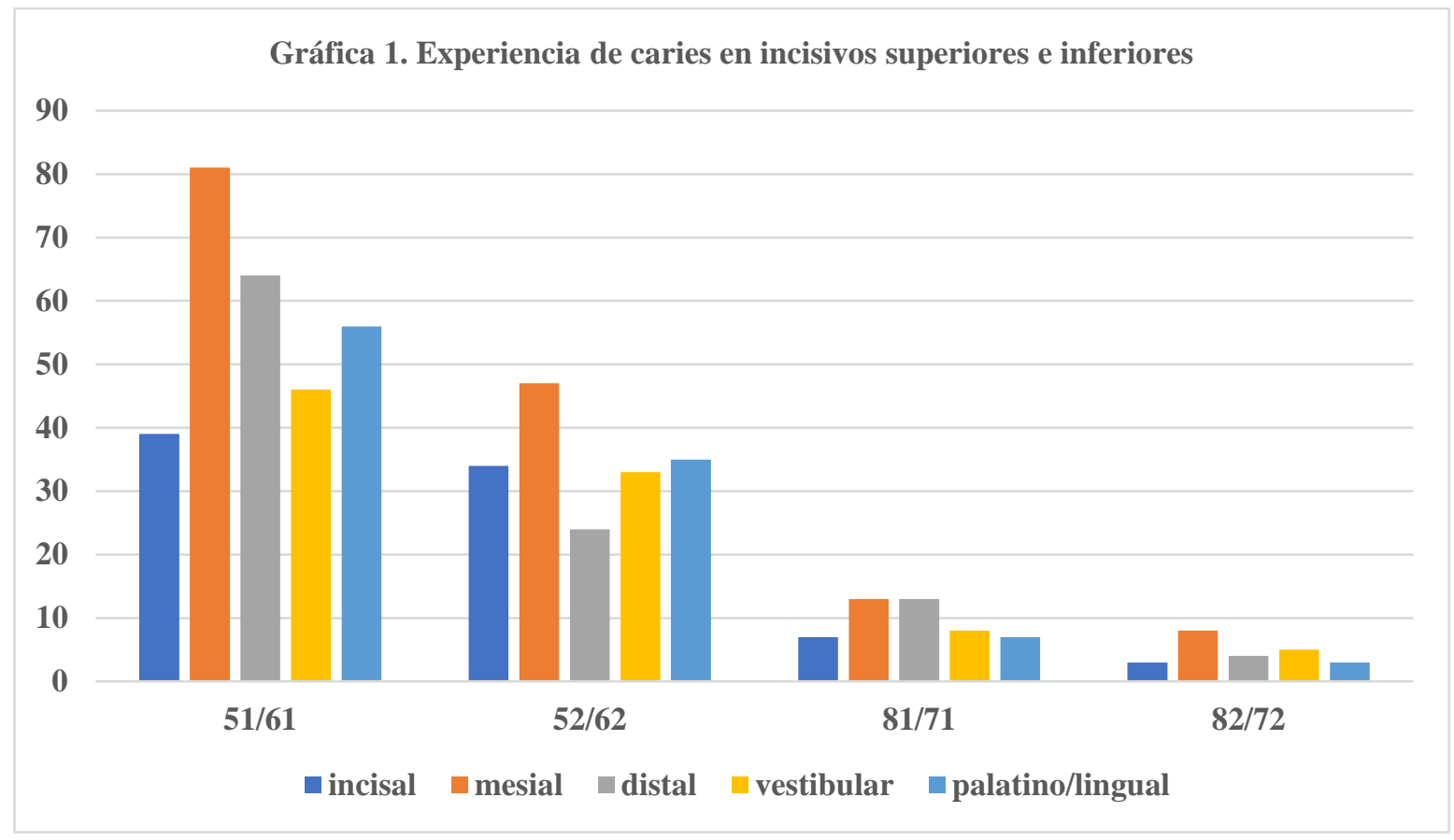

Al comparar las lesiones de caries en los caninos, la mayor presencia de lesiones se observó en los caninos superiores en todas sus caras y sobresale la cara vestibular en las superficies superiores y en los caninos inferiores en las superficies distales. Las superficies con menor afectación fueron las superficies mesiales (Gráfica 2).

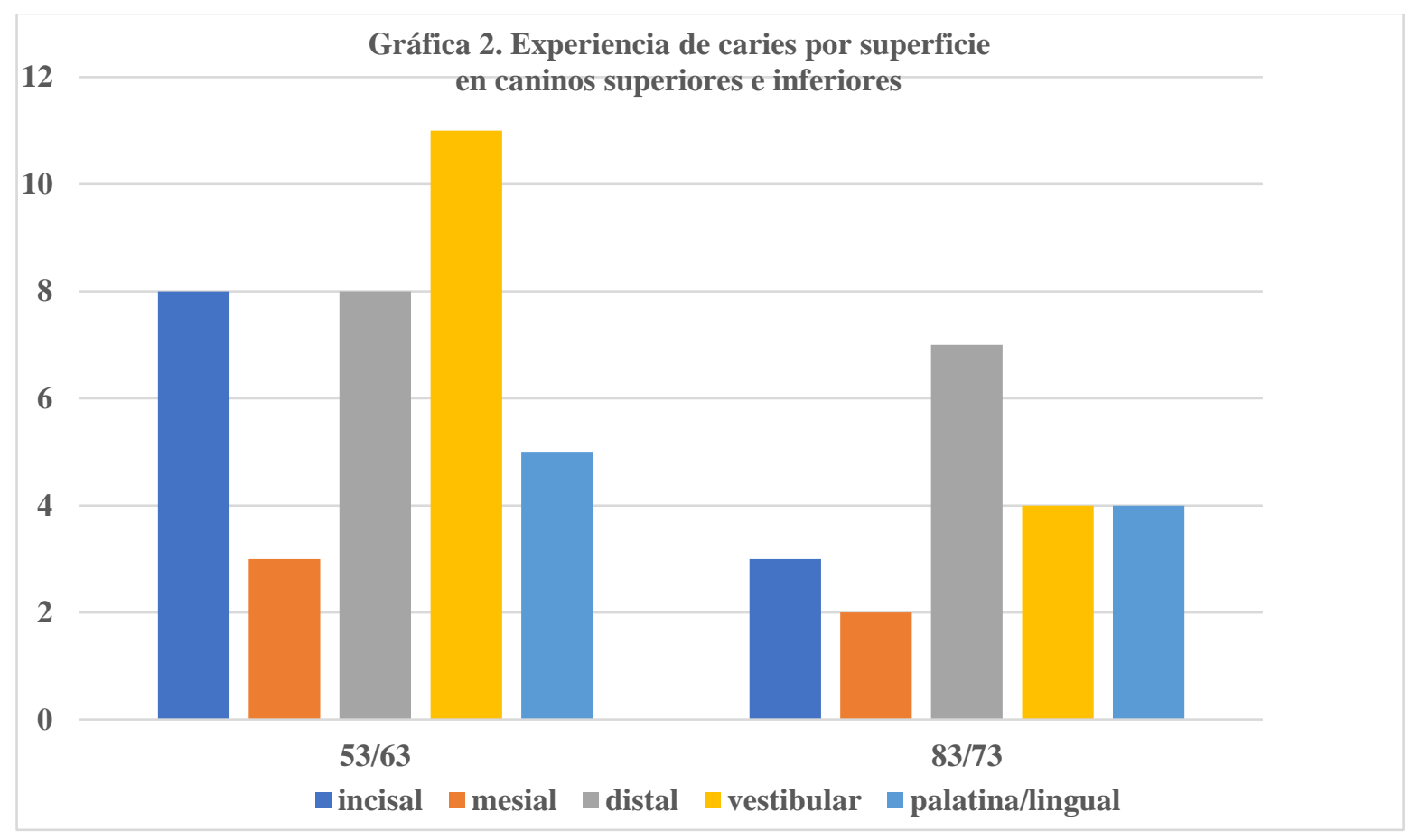


De todos los molares, los inferiores fueron los presentaron más lesiones de caries en la superficie oclusal, sobre todo los primeros molares (Gráfica 3).

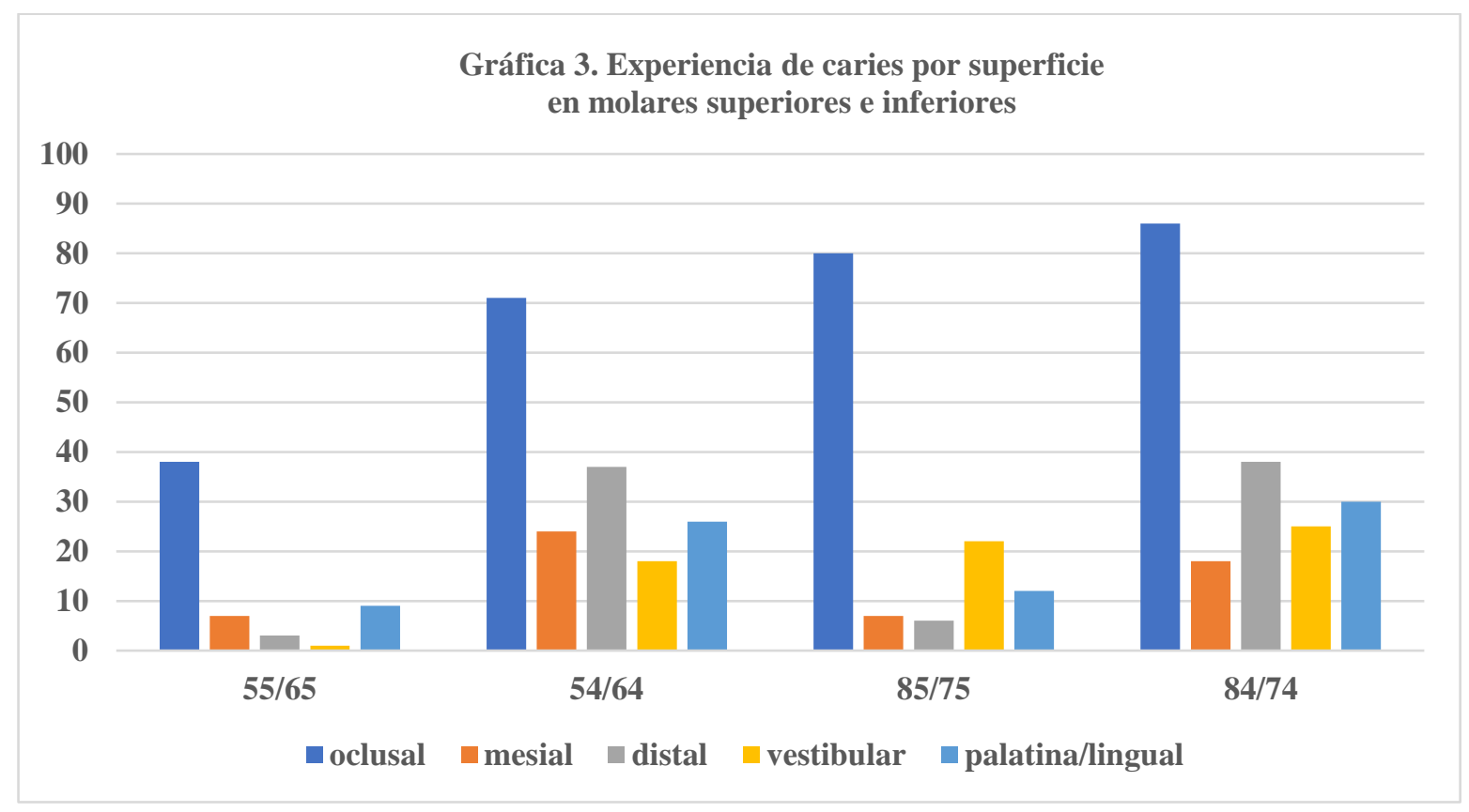

\section{DISCUSIÓN}

La prevalencia de CIT en el presente estudio fue de $41 \%$ a los 3 años, $79 \%$ a los 4 años y $62 \%$ a los 5 años, el índice cpos se incrementó conforme a la edad, sin diferencias entre sexos._Los dientes con mayor afectación fueron los incisivos centrales superiores y la superficie con más presencia de caries fue la oclusal de los primeros molares inferiores.

Si se compara la información de la prevalencia del CIT con datos del Sistema de Salud de México encontramos que los niños de 3, 4 y 5 años que asistieron a atención odontológica presentaron CIT de $64 \%, 74 \%$ y $76 \%$ respectivamente (Secretaría de Salud, 2020), esta prevalencia reportada es mayor que la observada en el presente estudio. La diferencia en la prevalencia de los niños de 3 años fue de un 23\% y la diferencia con los prescolares de 5 años, se pudo haber debido a una exfoliación de algunos dientes anteriores superiores y con ello una menor presencia de CIT en los preescolares estudiados.

Los resultados de CIT de la muestra estudiada, son mayores a una de las metas Globales de Salud Bucal propuesta por la OMS para el año 2000 (Global Goals for Oral Health in the year 2000, 1981), respecto a que el 50\% de niños entre 5 y 6 años estuvieran libres de caries, porcentaje no rebasado en las edades de 4 y 5 años. Esta meta fue alcanzada por países desarrollados desde la década de los 90’s, pero para una gran parte de la población mundial sigue siendo una aspiración lejana (Hobdell, Petersen, Clarkson y Johnson, 2003; Masood et al., 2018; Piovano et al., 2008). La OMS señala que más de 530 millones de niños sufren de caries en la dentición temporal (GBD, 2018), que se agudiza con la presencia 
de dolor, dificultades para comer, pérdida del sueño, ausencia escolar, problemas al realizar la higiene bucal (Achahui et al., 2014) y en general interferencia en las actividades diarias (Clarke et al., 2006; Nuttall y Harker, 2004). Esta morbilidad se podría reducir mediante intervenciones de salud pública dirigidas a factores de riesgo más comunes.

Se ha reportado en niños de 4 años en otros estados de México (Nayarit) (Guerrero et al., 2015) prevalencias del $50 \%$ y a los 5 años del $45 \%$, cifras menores a las del presente trabajo, tal vez debido a que dicho reporte corresponde a una zona con prevalencia de caries menor que la ciudad de México (Secretaría de Salud, 2014).

Al comparar la prevalencia del presente trabajo (62.5\%) con investigaciones de países latinos en edades de 3 a 5 años, las cifras reportadas para Colombia fueron de 35.4\% (Corchuelo y Soto, 2017), para Chile de 58\% (Echeverría, Henriquez, Sepúlveda y Barra, 2010) y para Perú de 89.4\% (Cayo, Cosme y Morales, 2019). Al desglosar por edades específicas la experiencia de caries, a la edad de 3 años en Colombia fue de 47.1\% (Ministro de Salud, 2014), a los 4 años en niños chilenos fue de 49.6\% (Ministerio de Salud, 2010) y a los 5 años para Brasil fue de 46.6\% (Ministerio da Salud, 2012), información que al comparar con la muestra analizada la prevalencia a la edad de 3 años fue de 40.7\%, a los 4 años de $78.9 \%$ y a los 5 años de $62 \%$ diferencias debidas probablemente, a los índices de caries de cada región, a las medidas de los servicios públicos de cada país y a los índices de desarrollo de las comunidades estudiadas.

Los resultados de esta investigación en los niños de 3 a 5 años muestran que tres de cada 5 niños presentó caries (62.5\%), prevalencia similar a lo reportado para Arabia Saudita (62.7\%) (Wyne, Ghannam, Shammery y Khan, 2002) y Perú (65\%) (Córdoba, María y Requejo, 2010) en edades similares y superior a países latinos como Colombia (Corchuelo y Soto, 2017) y Chile (Echeverría et al., 2010) con $35.4 \%$ y $58 \%$ respectivamente.

La presencia del cpos se fue incrementado conforme a la edad, datos menores que los reportados para otros estados del país, diferencias que probablemente se pueden deber, a una mayor prevalencia de caries para esos estados (Secretaría de Salud, 2001; Secretaría de Salud, 2014. Al analizar en ese mismo sentido el cpos a nivel internacional a la edad de 5 años en Argentina el índice fue más elevado $11.9 \pm 0.58$ (Piovano et al., 2008), esta diferencia puede estar condicionada a las medidas preventivas impuestas en el país (Estupiñán, 2005).

En todos estos estudios el componente cariado fue el que dominó, dato similar a este reporte y en relación al componente perdido en nuestra población, solo un niño a la edad de 3 años tuvo dos dientes perdidos y solo una niña a los 4 años tuvo un diente perdido.

Respecto a la mayor presencia de caries por grupo de dientes en los niños estudiados, el 39\% de la muestra presentó tanto en los dientes anteriores como en los posteriores, seguido de los dientes 
posteriores (16.2\%) y con CIT sólo en dientes anteriores el 7.4\% de los niños. Para el primer grupo de dientes afectados la presencia de uno de los factores de riesgo pudo ser la anatomía dental básicamente remarcada por las fosetas y fisuras de los dientes posteriores que favorecen la formación de nichos ecológicos y con ello el desarrollo de caries y para los dientes anteriores la presencia frecuente de ingesta de líquidos con contenido de hidratos de carbono naturales como la maltosa o fructuosa, o a los que se les agrega en muchas ocasiones azúcar, que sumada a la falta de higiene, favorecen la formación de lesiones cariosas. Este mismo patrón ha sido reportado en molares y dientes anteriores con el 50.3\%, sólo en posteriores $42.2 \%$ y en anteriores $7.5 \%$ aunque con valores más altos en los primeros grupos (Wyne, 2008).

La CIT afectó en primer lugar a los incisivos centrales superiores de ambos lados, debido probablemente a la exposición frecuente que tienen estos dientes con alimentos cariogénicos que se agrava ante la ausencia de higiene, siguieron en afectación los primeros molares inferiores y superiores probablemente por las características anatómicas retentivas que favorecen el desarrollo de los microorganismos y con ello la desmineralización del esmalte, así como por haber erupcionado casi un año antes que los segundos molares.

Otros investigadores del medio oriente (Wyne et al., 2002) reportan prevalencias más altas para los molares inferiores, seguidos de los incisivos centrales superiores afectando sobre todo la cara mesial, con un patrón por superficie similar al de la presente investigación. Estas diferencias pueden estar condicionadas por los reportes de altas prevalencias de caries en la región (Al Mughery, Attwood y Blinkhorn, 1991; Janson y Fakhouri, 1993; Wyne, et al., 1996), de $72 \%$ a $78 \%$.

Si analizamos el comportamiento de CIT por superficie de cada diente, es importante señalar que el primer molar inferior fue el más afectado de todos los molares en todas sus caras excepto en la cara mesial, que fue menor debido probablemente a la presencia del espacio primate entre la cara distal del canino y la mesial del primer molar.

Respecto a los segundos molares la presencia de caries fue más evidente en los inferiores y principalmente en las superficies oclusales y vestibulares, una de las superficies con menor presencia de caries tanto en los inferiores como en los superiores fue la superficie distal, probablemente porque no hay contacto proximal, lo que disminuye el riesgo de desarrollar lesión cariosa.

Otros autores reportan para niños de 4 a 6 años en un estudio longitudinal (Cortes, Ekstrand y Martignon, 2018), que el mayor daño observado fue en primeros molares inferiores en las caras oclusales y distales, seguidos por los segundos molares inferiores en las caras oclusales y mesiales, similar a lo reportado en nuestro estudio.

En el comportamiento de caries en el grupo de los incisivos en la muestra estudiada, se encontró un mayor daño en los incisivos superiores (centrales y laterales). El patrón de afectación por superficie 
de los incisivos, correspondió a la cara mesial para los centrales superiores y menor en la superficie distal para los laterales, probablemente por el espacio primate entre este diente y el canino.

Respecto a los incisivos inferiores la cara distal de los centrales fue la más afectada y para los laterales la cara mesial y las menos afectadas en todos estos dientes fueron las caras incisales y linguales, debido probablemente a la presencia del flujo salival contante proveniente principalmente de las glándulas salivales sublinguales.

La presencia de caries en el grupo de los caninos fue mayor en los caninos superiores principalmente en las superficies vestibulares e incisales, las caras con menor presencia de lesiones cariosas correspondieron a la cara mesial, que para el caso del canino superior se pudo deber a la presencia del espacio primate que facilita la higiene bucal y la autoclisis.

Todo lo expuesto evidencia la importancia de la prevención en la estomatología, que busca la conservación de la integridad de la dentición y como consecuencia una mejor salud general. Cobra relevancia conocer las condiciones de salud bucal de los niños e implementar, la identificación de factores de riesgo centrados en el diagnóstico, que lleven a la detección y control de enfermedades bucales en etapas tempanas a través de la promoción de la salud y acciones de protección específica de mayor eficacia como lo es la aplicación del barniz fluorado y/o de selladores de fisuras y fosetas.

\section{CONCLUSIONES}

La prevalencia global de CIT fue de $62.5 \%$.

- Los dientes más afectados por caries dental fueron los incisivos centrales superiores, seguidos de los primeros molares inferiores.

- Las superficies dentales con mayor afectación fueron las oclusales de los primeros molares inferiores seguidas de las caras mesiales de los incisivos centrales superiores.

- Conforme la edad de los preescolares avanza, independientemente del sexo, el porcentaje de dientes cariados aumenta, lo que conlleva a tratamientos invasivo-restauradores a edades muy tempranas. 


\section{REFERENCIAS}

- $\quad$ Achahui, A. P., Albinagorta, M. J., Arauzo, S. C. J., Cadenillas, S. A. M., Céspedes, M. G. P., Cigüeñas, R. E. M., Díaz, H. S. A., Olano, V. Z. G., Pinto, M. C., Paredes, Q. C., y Julca, S. G. (2014). Caries de infancia temprana: diagnóstico e identificación de factores de riesgo. Revista Odontología Pediátrica, 13(2), 119-137. http://op.spo.com.pe/index.php/odontologiapediatrica/article/view/108

- $\quad$ Al Mughery, A. S., Attwood, D., y Blinkhorn, A. S. (1991). Dental health of 5-years-old children in Abu Dhabi, United Arab Emirates. Community Dent Oral Epidemiol, 19, 308-309. https://pubmed.ncbi.nlm.nih.gov/1742999/

- $\quad$ American Academy of Pediatric Dentistry. (2003). Oral health polices and clinical guidelines. Pediatric Dentistry, 25(7 Suppl.), 11-49.

- $\quad$ American Academy of Pediatric Dentistry. (2008-2009). Policy for early childhood caries (ECC): Classifications, consequences and preventive strategies. Pediatr Dent, 30 suppl. 7:40-43.

- $\quad$ Begzati, A., Berisha, M., y Meqa, K. (2010). Early childhood caries in preschool children of Kosovo a serious public health problem. BMC Public Health, 10, 788. https://bmcpublichealth.biomedcentral.com/articles/10.1186/1471-2458-10-788

- $\quad$ Bravo, P. M., Almerich, S. J. M., Ausina, M. V., Avilés, G. P., Blanco, G. J. M., Canorea D. E., Casals, P. E., Gómez, S. G., Hita, I. C., Llodra C. J. C., Monge, T. M., Montiel, C. J. M., Palmer, V. P. J., y Sainz, R. C. (2016). Encuesta de Salud Oral en España 2015. RCOE, 21 (Supl. 1), 8-48. https://www.consejodentistas.es/comunicacion/actualidad-del-consejo/publicaciones-delconsejo/revistas-del-consejo/revista-rcoe/item/1238-encuesta-de-salud-oral-en-espana-2015-revistarcoe-junio-2016-vol-21-suplemento-1.html

- $\quad$ Cayo, T. N. V., Cosme, R. A. T., y Morales, V. R. (2019). Caries dental y su relación con el nivel de conocimiento sobre salud bucal de los padres de familia en preescolares. Kiru 16(3), 102-107. https://www.aulavirtualusmp.pe/ojs/index.php/Rev-Kiru0/article/view/1597

- $\quad$ Clarke, M., Locker, D., Berall, G., Pencharz, P., Kenny, D. J., y Judd, P. (2006). Malnourishment in a population of young children with severe early childhood caries. Pediatr Dent, 28, 254-259. https://pubmed.ncbi.nlm.nih.gov/16805358/

- $\quad$ Corchuelo, O. J., y Soto, L. L. (2017). Prevalencia de caries en preescolares de hogares comunitarios en el Valle del Cauca y factores sociales relacionados. Revista Odontológica Mexicana. Vol. 21, Núm. 4 Octubre-Diciembre, 229-234. http://www.scielo.org.mx/scielo.php?script=sci_arttext\&pid=S1870-199X2017000400229

- Córdoba, S., D., María, C., F., y Requejo, B., A. (2010). Caries dental y estado nutricional en niños de 3 a 5 años de edad. Chiclayo, Perú, 2010. Kiru, 7(2), 57-64. https://www.usmp.edu.pe/odonto/servicio/2010/Kiru2010v7n2/Kiru2010v7n2art2.pdf

- $\quad$ Cortes, A., Ekstrand, K. R., y Martignon S. (2018). Visual and radiographic merged-ICDAS caries progression pattern in 2-6 years old Colombian children: Two-year follow-up. Int J Paediatr Dent Nov 15. doi: 10.1111/ipd.12448. Epub ahead of print. PMID: 30431189. 
- $\quad$ Echeverría, L. S, Henriquez, D. E., Sepúlveda, R. R., y Barra, P. M. (2010). Caries temprana de la infancia severa: impacto en la calidad de vida relacionada a la salud oral en niños preescolares. Revista Dental de Chile 101(2), 15-21).

- $\quad$ Estupiñán, D. S. (2005). Promoting Oral Health. The Use of Salt Fluoridation to Prevent Dental Caries. Pan American Oral Health Organization. Washington D. C. PAHO. https://pdfdrive.com/pdf/Pan20American20Health20Organization2020Promoting20Oral20Health.20the20Use20of20Salt20Fluoridation20to20Prevent20Dental20Caries20 28PAHO20Scientific20Publications29.pdf

- $\quad$ Featherstone, J. D., Adair, S., M., Anderson, M. H., Berkowitz, R. J., Bird, W. F., Crall, J. J., Den Besten, P. K., Donly, K. J., Glassman, P., Milgrom, P., Roth, J. R., Snow, R., y Stewart, R. E. (2003). Caries management by risk assessment: consensus statement. J Calif Dental Assoc, 31(3), 257269. https://pubmed.ncbi.nlm.nih.gov/12693825/

- $\quad$ Fejerskov, O., Nyvad, B., y Kidd, E. (2015). Dental caries. The disease and its clinical management. 3rd ed. U. S.: WILEY Blackwell.

- GBD 2016 Disease and Injury Incidence and Prevalence Collaborators. (2017). Global, regional, and national incidence, prevalence, and years lived with disability for 328 diseases and injuries for 195 countries, 1990-2016: a systematic analysis for the Global Burden of Disease Study 2016. Lancet, 390, 1211-1259. https://doi.org/10.1016/S0140-6736(17)32154-2.

- Global Goals for Oral Health in the Year 2,000. (1981). Federation Dentair International. 32(1), 74-77.

- $\quad$ GBD 2017 Disease and Injury Incidence and Prevalence Collaborators (2018). Global, regional, and national incidence, prevalence, and years lived with disability for 354 diseases and injuries for 195 countries and territories, 1990-2017: a systematic analysis for the Global Burden of Disease Study 2017. Lancet, 392: 1789-1858. https://www.sciencedirect.com/science/article/pii/S0140673618322797

- $\quad$ Guerrero, C., M., P., Aguilar, F., E., G., Gutiérrez, R., J., F., y Lara, S., I., M. (2015). Prevalencia de caries temprana de la infancia y necesidad de tratamiento en niños que asisten a la clínica de la especialidad en Odontopediatría de la Universidad Autónoma de Nayarit. Rev. Latinoamericana de Ortodoncia y Odontopediatría, https://www.ortodoncia.ws/publicaciones/2015/art-14/

- $\quad$ Hobdell, M., Petersen, P. E., Clarkson, J. y Johnson, N. (2003). Global goals for oral health 2020. Int Dent J, 53, 285-288. https://www.who.int/oral_health/media/en/orh_goals_2020.pdf

- Instituto Nacional de Estadística y Geografía. (2011). Distrito Federal. Principales resultados por localidad». XII Censo de Población y Vivienda. México.

- Instituto Nacional de Estadística y Geografía. (2020). Distrito Federal. Principales resultados por localidad». XII Censo de Población y Vivienda. México.

- Janson, S., y Fakhouri, H. (1993). Dental health in suburban Jordanian preschool children. Swed Dent J, 17, 123-127. https://europepmc.org/article/med/8356536 
- Kagihara, L., E., Niederhauser, V., P., y Star, M. (2009). Assessment, management, and prevention of early childhood caries. $J$ Am Acad Nurse Pract. 21(1), 1-10. https://onlinelibrary.wiley.com/doi/full/10.1111/j.1745-7599.2008.00367.x

- $\quad$ Kassebaum, N., J., Bernabe, E., Dahiya, M., Bhandari, B., Murray, C., J., y Marcenes, W. (2015 May). Global burden of untreated caries: a systematic review and metaregression. J Dent Res, 94(5), 650-658.

https://scholar.google.com.mx/scholar?hl=es\&as_sdt=0\%2C5\&as_vis=1\&q=Global+burden+of+untre ated+caries $\% 3 \mathrm{~A}+\mathrm{a}+$ systematic+review+and+metaregression. $+\& \mathrm{btnG}=$

- $\quad$ Marshall, T., A., Levy, S., M., Broffitt, B., Warren, J., J., Elchenberger-Gilmore, J., M., Burns, T., L., y Stumbo, P., J. (2003). Dental Caries and Beverage Consumption in Young Children. Pediatrics, 112(3), 184-191.

- $\quad$ Masood, M., Mnatzaganian, G., y Baker, R. S. (2018). Inequalities in dental caries in children within the UK: Have there been changes over time? Community Dent Oral Epidemiol. Feb; 47(1), 7177. doi: 10.1111/cdoe.12426. Epub Oct 9. https://pubmed.ncbi.nlm.nih.gov/30298932/

- $\quad$ Ministério da Saúde. (2012). Pesquisa Nacional de Saúde Bucal: resultados principais. Secretaria de Atenção à Saúde. Secretaria de Vigilância em Saúde. Brasília.

- Ministerio de Salud. (diciembre 2010). Análisis de situación de salud bucal en Chile. Subsecretaría de Salud Pública División Prevención y Control de Enfermedades Departamento Salud Bucal. Chile.

- $\quad$ Ministro de Salud y Protección Social. (2014). IV Estudio Nacional de Salud Bucal. Situación de Salud Bucal. Colombia.

- Norma Oficial Mexicana 040. (2010, 2 de febrero). NOM-040-SSA1-1993, Productos y Servicios. Sal Yodada y Sal Yodada Fluorurada. Especificaciones Sanitarias. Diario Oficial de la Federación. México. http://legismex.mty.itesm.mx/normas/ssa1/ssa1040-Mod2010_02.pdf

- $\quad$ Nuttall, N., y Harker, R. (2004). Impact of Oral Health. Children's Dental Health in the United Kingdom 2003. London, UK: The Stationery Office.

- $\quad$ Peres, M., A., Dias de Oliveira, L., M., R, Sheiham, A., Glazer, P., K., Barros, F., C., Gonzales, H., P., Nunes, M., A., M., Romano, A., R., y Gomes, V., C. (2005). Social and biological early life influences on severity of dental caries in children aged 6 years Community Dent Oral Epidemiol, 33(1), 53-63.

- $\quad$ Petersen, P. (2003). The world oral health report 2003: continuous improvement of oral health in the 21 st century - the approach of the WHO Global Oral Health Programme. Community Dent Oral Epidemiol, 31, 3-23.

- $\quad$ Piovano, S., H., Bordoni, N., Doño, R., Ángela, B., Argentieri, A., B., Cohen, A., S., Klemonskis, G., L., Macucho, M., E., P.,edemonte, Z., A., Pistochini, A., B., y Squassi, A., F. (2008). Estado dentario en niños, adolescentes y adultos de la Ciudad Autónoma de Buenos. Rev. Fac. Odontol, 23(54/55), 34 42. 
- $\quad$ Programa de Naciones Unidad para el Desarrollo. (2015). Índice de Desarrollo Humano Municipal 2010 N. M. https://es.wikipedia.org/wiki/Ciudad_de_M\%C3\%A9xico\#cite_ref-132

- $\quad$ Rodríguez, V., L., E., Contreras, B., R., Arjona, S., J., Soto, M., M., R., y Tavira, J., A. (2006). Prevalencia de caries y conocimientos sobre salud-enfermedad bucal de niños ( 3 a 12 años) en el Estado de México. Rev ADM. LXIII, (5), 170-175.

- Secretaría de Salud. (2001). Encuesta Nacional de Caries Dental. Programa de Salud Bucal. México.

- $\quad$ Secretaría de Salud. (2014). Encuesta Nacional de Caries y Fluorosis Dental 2011-2014. CENAPRECE. México

- $\quad$ Secretaría de Salud. (2020). Sistema de Vigilancia Epidemiológica de Patologías Bucales SIVEPAB 2019. México. https://www.gob.mx/cms/uploads/attachment/file/597944/resultadosSIVEPAB_2019.pdf

- Sheiham, A. (2006). Dental caries effects body weight, growth and quality of life in pre-school children. British Dental Journal, 201(10), 625-626. DOI:10.1038/sj.bdj.4814259

- $\quad$ Villena, S., R., Pachas, B., F., Sánchez, H., Y., y Carrasco, L., M. (2011). Prevalencia de caries de infancia temprana en niños menores de 6 años, residentes en poblados urbano-marginales de Lima Norte. Rev. Estomatol Herediana, 21(2), 79-86.

- $\quad$ World Health Organization. (2013). Oral Health Surveys. 5a ed. Geneva, Switzerland.

- $\quad$ Wyne, A., Darwish, S., Adenubi, J., Battata, S., y Khan N. (1996). Caries prevalence and pattern in Saudi preschoolers. J Dent Res, 75, 360. Abstract 2739.

- Wyne, A., H., Al-Ghannam, N., A., Al-Shammery, A., R., y Khan, N., B. (2002). Caries prevalence, severity and pattern in preschool children. Saudi Med J, 23:580-584.

- Wyne, A., H. (2008). Caries prevalence, severity, and pattern in preschool children. J Contemp Dent Pract. 9(3), 24-31. 